Gesnerus 54 (1997) 258-267

\title{
News and Activities
}

\section{Prof. Loris Premuda zum 80. Geburtstag}

Das Ehrenmitglied der Schweizerischen Gesellschaft für Geschichte der Medizin und der Naturwissenschaften (SGGMN), Loris Premuda, konnte am 4. Januar dieses Jahres seinen 80 . Geburtstag begehen.

Loris Premuda wurde 1917 im damals österreichischen Montana auf der Halbinsel Istrien geboren. Aus diesen geographischen sowie auch familiären Gründen verbanden sich in Premuda italienische und deutsche Kultur und Sprache, sehr zum Gewinn seines medizinhistorischen Schaffens. Nach dem Studium der Medizin an der Universität Padua war Premuda während der letzten Jahre des Zweiten Weltkriegs beim Internationalen Roten Kreuz tätig, sodann an Kliniken in Zürich, Wien und Triest.

1948 habilitierte er sich für Geschichte der Medizin an der Universität Rom. Sein weiterer Weg führte über Ferrara 1954 an die Universität Padua, wo dem Forscher der Aufstieg in die Spitzengruppe der Medizingeschichte gelang und wo er 1956 ein Institut für Geschichte der Medizin gründete und 1968 zum Ordinarius für das Fach ernannt wurde.

Premudas wissenschaftliches Werk ist von einer aussergewöhnlichen Breite gekennzeichnet. Ausser in Büchern und Monographien hat Premuda die grosse Fülle seiner Themen in einigen hundert Zeitschriftenartikeln und in Sammelwerken behandelt. Neben der Medizin in Padua und Triest veröffentlichte er Studien zur antiken Medizin, zur Geschichte der Psychosomatik, der Pädiatrie, der Quarantäne, der Pharmazie, einzelner Krankheitsbilder sowie Biographien.

Premuda hat viele Ämter bekleidet und Ehrungen empfangen. Die Verleihung der Ehrenmitgliedschaft in der SGGMN erfolgte in erster Linie in Würdigung seiner Verbindung mit dieser Gesellschaft und seiner vielfältigen Beziehungen zur Schweiz.

Zu Beginn dieses Jahres hielt der Achtzigjährige - immer noch frisch und fesselnd - am Medizinhistorischen Institut der Universität Bern einen Vortrag zum Thema «Padua und die Medizin». Die Redaktion freut sich, eine schriftliche Fassung davon als ersten Artikel dieses Heftes abzudrucken, und 
sie gratuliert damit Professor Premuda und wünscht ihm neben verdienter Beschaulichkeit weitere erfüllende Aktivitäten.

M. H. B.

\section{Prof. Dr. Marcel H. Bickel zum 70. Geburtstag}

Am 14. Oktober 1997 feierte Prof. Dr. phil. nat. Marcel Henry Bickel, Chefredaktor des Gesnerus, seinen 70. Geburtstag. Der Vorstand der Schweizerischen Gesellschaft für Geschichte der Medizin und der Naturwissenschaften (SGGMN) gratuliert ihm dazu herzlich und dankt ihm für seine grosse und erfolgreiche Arbeit.

Nach dem Studium der Chemie und Biochemie an der Universität Basel bildete er sich in Rom bei D. Bovet und in Bethesda/USA bei B. B. Brodie zum Pharmakologen aus und wurde 1967 Privatdozent und Extraordinarius, 1971 Ordinarius ad personam am Medizinisch-chemischen Institut der Universität Bern. 1977 erhielt er die Berufung zum Ordinarius für Pharmakologie und zum Direktor des Berner Pharmakologischen Instituts, in dem er bis zur Emeritierung 1993 tätig war. Seine langjährige Forschungsarbeit über Metabolismus und Kinetik von Medikamenten, über Psychopharmakologie und Toxikologie wurde u. a. 1994 mit dem Ebert Prize der American Pharmaceutical Association und 1996 mit der Einladung nach London zur King's College Lecture in Pharmaceutical Sciences geehrt.

Mit Henry E. Sigerist als Onkel hatte Marcel H. Bickel bereits als Gymnasiast das Glück, erleben zu können, was Medizingeschichte ist und wie sie auf höchstem Niveau betrieben wird. Auch als belasteter Pharmakologe nahm er sich Zeit für bedeutende historische Studien; erwähnt seien seine Arbeiten über Marceli Nencki und über die Geschichte des Medikamentenmetabolismus (mit C. Bachmann und A. Conti). Als Präsident mehrerer fakultärer Kommissionen erreichte er die Institutionalisierung und Konsolidierung des Fachs Medizingeschichte an der Universität Bern.

Der Schweizerischen Gesellschaft für Geschichte der Medizin und der Naturwissenschaften (SGGMN) stellte Marcel H. Bickel seine Dienste mehrfach zur Verfügung, so als Präsident in den Jahren 1987 bis 1989 und als Präsident der Dr. Markus Guggenheim-Schnurr-Stiftung seit 1992. Seit der Emeritierung 1993 widmet er sich neben seinem Amt als Chefredaktor des Gesnerus ganz der Erforschung und Darstellung der Geschichte der Pharmakologie. Im Berner Medizinhistorischen Institut, wo seine «Redaktions- 
stube» eingerichtet ist, ist Marcel H. Bickel als freier Mitarbeiter, als vielseitig erfahrener Ratgeber und Helfer und als verlässlicher, grosszügiger Kollege hoch geschätzt.

Herzliche Glückwünsche, und: Ad multos annos!

Urs Boschung, Präsident SGGMN

\section{Verleihung des Conrad Ferdinand Meyer-Preises 1996: Auszeichnung für Medizinhistoriker Christoph Mörgeli}

Der Conrad Ferdinand Meyer-Preis wird alljährlich an einen oder zwei jüngere, im Kanton Zürich niedergelassene Gelehrte, Künstler und Schriftsteller verliehen.

Die mit einer Zuwendung verbundene Preisverleihung erfolgt seit 1937 durch den Stiftungsrat der von Camilla Meyer auf ihr Ableben hin zum Andenken an ihren Vater, den Dichter Conrad Ferdinand, errichteten gemeinnützigen Conrad Ferdinand Meyer-Stiftung in Zürich. Privatdozent Christoph Mörgeli erhält für sein wissenschaftliches Schaffen auf medizinhistorischem Gebiet den Conrad Ferdinand Meyer-Preis 1996. Er hat mit seiner Dissertation über den Arzt, Wissenschaftler und Staatsmann Johannes Hegetschweiler und mit seiner Habilitationsschrift über die europäische Medizin der Biedermeierzeit, erschlossen aus den Reiseberichten des Arztes Conrad Meyer-Hofmeister, zwei medizinhistorische Arbeiten von hoher wissenschaftlicher Qualität vorgelegt, die einen engen Bezug zu Stadt und Kanton Zürich haben. Als Konservator am Medizinhistorischen Museum leistet er hier in Zürich, in einer heute so wichtigen Brückenfunktion zwischen Naturund Geisteswissenschaften, eine Arbeit, die für Kultur, Wissenschaft und Bildung von hoher Bedeutung ist, was sich auch in der grossen Beachtung zeigt, welche die Ausstellungen des Museums finden. Dass Christoph Mörgelis Tätigkeit auch internationale Ausstrahlung hat, ist unter anderem 1994 in der Durchführung des europäischen Kongresses der medizinhistorischen $\mathrm{Mu}$ seen in Zürich zum Ausdruck gekommen.

Elvira Parietti-Bachmann 


\section{Ehrung für Alfred Schett}

Anlässlich des 80. Geburtstages überreichte am 27. März 1997 an einer kleinen Feierstunde ein Vertreter des Kuratoriums der sehr angesehenen AlfredVogt-Stiftung zur Förderung der Augenheilkunde (Zürich) dem St. Galler Forscher Alfred Schett eine Anerkennungsgabe. Sie würdigt damit das bedeutende wissenschaftliche Werk eines ungewöhnlichen Forschers.

Alfred Schett, ein pensionierter Elektroingenieur, beschäftigte sich schon früh mit dem Mikroskopieren. Daraus entwickelte sich ein Interesse für Mikroskope und später dann auch für den Augenspiegel, eines der wichtigsten Untersuchungsinstrumente in der Augenheilkunde. Intensiv widmete sich Alfred Schett dessen Geschichte und legte auch eine private Sammlung solcher Instrumente an. Aus der Beschäftigung mit ihnen ergab sich 1982 ein Kontakt mit dem damaligen Konservator des Medizinhistorischen Museums der Universität Zürich. In der Folge ordnete und bearbeitete Alfred Schett auch die Sammlung von Augenspiegeln und verwandten Instrumenten des Zürcher Museums wissenschaftlich und berichtete darüber 1990 in der Publikation «Vom Helmholtz-Augenspiegel zur Funduskamera» (Gesnerus Supplement Nr. 41). Nach weiterer jahrelanger intensiver Forschungsarbeit an Hunderten von Originalen auch aus ausländischen medizinhistorischen Sammlungen und grossem Literaturstudium ist nun von Alfred Schett vor kurzem eine umfangreiche Monographie ersten Ranges mit dem Titel «Der Augenspiegel, ein Beitrag zur Entwicklungsgeschichte bis zum Beginn des 20. Jahrhunderts» (mit 330 Abbildungen) im Verlag J.P. Wayenborgh, Ostende erschienen.

Peter Speiser

\section{Amerikanischer Wissenschaftshistoriker erhält in Darmstadt den Paul-Bunge-Preis 1997}

Der Paul-Bunge-Preis zur Historie wissenschaftlicher Instrumente der Hans R. Jenemann-Stiftung, getragen von der Gesellschaft Deutscher Chemiker (GDCh) und der Deutschen Bunsen-Gesellschaft für Physikalische Chemie (DBG), wurde anlässlich der Bunsen-Tagung 1997 (DBG-Hauptversammlung) am 9. Mai in Darmstadt an Dr. Silvio A. Bedini, Washington, verliehen. Von der Stiftung werden aus gleichem Anlass zwei Nachwuchswissenschaftler ausgezeichnet.

Die Vergabe des Paul-Bunge-Preises an Bedini erfolgt in Anerkennung eines beeindruckenden wissenschaftlichen Lebenswerkes, das die gesamte 
Spannweite moderner Instrumentengeschichte umfasst und die Erforschung der material culture of science entscheidend vorangebracht hat.

Bedini, 1917 in Ridgefield, Connecticut, geboren, studierte an der Columbia University und war bis 1987 in leitenden Positionen am National Museum of History and Technology, Smithsonian Institution, in Washington tätig. Unter seinen zahlreichen wissenschaftlichen Veröffentlichungen ist eines der jüngsten und bemerkenswertesten Werke das 1994 erschienene Buch «Science and Instruments in Seventeenth-Century Italy». Bedini, Mitglied mehrerer amerikanischer und europäischer wissenschaftlicher Gesellschaften, arbeitet nach wie vor an umfassenden wissenschaftlichen Veröffentlichungen, wie z.B. zum Thema «The Vatican and Science». Der Paul-BungePreis ist mit 10000 Mark dotiert.

Für ihre herausragende, im vergangenen Jahr vorgelegte Magisterarbeit «Die feinmechanische Werkstatt von Rudolf Fuess - Eine Studie zur Zusammenarbeit von Handwerk und Wissenschaft am Beispiel des Assmannschen Aspirationspsychrometers von 1887 bis 1891» wird Claudia SchusterSpiekenheier aus Berlin ausgezeichnet. Jan Frercks aus Oldenburg fertigte im vergangenen Jahr eine herausragende Diplomarbeit über «Die Zahnradmethode zur Messung der Lichtgeschwindigkeit - Replikation von Fizeaus Experiment» an, die ebenfalls in Darmstadt ausgezeichnet wird. Beide Nachwuchswissenschaftler werden mit je 5000.- DM gefördert.

\section{Internationale Arbeitsrunde zur Geschichte der Seelenheilkunde}

\section{Tagungsbericht:}

Zum ersten Mal in ihrer Geschichte traf sich 1997 die «Internationale Arbeitsrunde zur Geschichte der Seelenheilkunde» ausserhalb Deutschlands. Die Tagung in Rom in der «Accademia Lancisiana» vom 29.5.97-30.5.97 hatte «Krankheitsbilder - Krankheitskonzepte» in der Geschichte der Seelenheilkunde zum Thema und wurde in Kooperation mit dem Auslandsreferat für Italien der DGPPN (R. Schmidt, Konstanz, und I. Hinnental Mandelli, Köln) und der Italienischen Gesellschaft für Psychiatrie (Prof. Munizza, Turin) veranstaltet. Das Goethe-Institut in Rom (A. Fackler-Kabisch) hatte freundlicherweise die Schirmherrschaft übernommen. Die «Accademia Lancisiana», nach dem gebürtigen Römer und Leibarzt dreier Päpste Giovanni Maria Lancisi (1654-1720) benannt, hat sich im besonderen mit der Geschichte der Medizin beschäftigt.

Professor Piccione, ärztlicher Leiter des «Dipartimento di Salute Mentale Roma E», führte zu Beginn der Tagung in das aktuelle Gesundheitssystem 
der psychiatrischen Versorgung in Italien und Rom ein. Die seit wenigen Jahren angelaufene Zeitspanne der Entwicklung wurde von ihm als «Phase der seelischen Gesundheit» bezeichnet. Mit Stolz berichtete er von der Auflösung aller psychiatrischen Krankenhäuser im Rahmen der italienischen Psychiatriereform. Nach insistierendem Nachfragen wurde offenbar, dass in Italien noch 3 psychiatrische Krankenhäuser existieren.

Die Tagungsthemen waren als «Gang durch die historische Entwicklung» angelegt. J. Wilmans begann deshalb mit «Seelische Störungen aus der Sicht griechischer und römischer Ärzte. Krankheitstheorien und Therapieversuche». J. Glatzel stellte die weitergehende wirksame Überlieferung antiken Denkens an Hand des Themas «Die Nobilitierung des Wahnsinns - die Tradition des aristotelischen Melancholieverständnis» vor. Das Jahrtausende lange Fortwirken der Galenischen Denktradition wurde von M. Kutzer demonstriert: «Da die Geistseele immateriell und in ihrer Essenz unveränderlich ist... Die galenistische Konzeption psychischer Krankheit im 16. und 17. Jahrhundert». R. Appell beschrieb einen konzeptionellen Sonderweg, der die Homöopathie begründete: «S. Hahnemann und die Anwendung des Ähnlichkeitsdenkens auf die Psychiatrie seiner Zeit». Bezüge zur zeitgenössischen Psychiatrie und zur Gegenwart fand E. Quinkler-Koch in einem literarischen Beispiel für den Selbstheilungsversuch einer Wahnsinnigen, dargestellt von Goethe in seinem Stück «Lila». Der Vortrag von H. Gröger: «Der paradigmatische Wandel in der Wiener medizinischen Schule der Psychiatrie des ausgehenden 19. Jahrhunderts - von der anatomischen Hirnforschung (Meynert) zur klinischen Phänomenologie (Krafft-Ebing)» musste aus Krankheitsgründen leider entfallen. Das ursprünglich von A. Homberg und F. Fagioli geplante Referat «Zur Einführung der medizinischen Methodik in die Psychiatrie: die Hysterie» wurde ersetzt durch die Darstellung der Bedeutung von Bildern in der Konstruktion von psychischer Wirklichkeit. N. Lalli beklagte in seiner Darstellung der «Psychotherapie in Italien vom Beginn des 20. Jahrhunderts bis heute» die deprimierende Situation, in der sich die Psychotherapie durch die zunehmende Biologisierung der Psychiatrie in Italien befinde. F. Giacanelli zeigte in seinem Vortrag «Krankheitsmodelle und Ideologien der italienischen Psychiatrie zwischen den beiden Weltkriegen» auf, dass auch die italienische Psychiatrie rassehygienischen und eugenischen Konzeptionen gegenüber empfänglich war. Ob planmässige Tötungen von Geisteskranken in Italien vorgekommen sind, konnte wegen fehlender Untersuchungen nicht sicher nachgewiesen werden. Faschisten hätten in Norditalien im Jahre 1943 einige Geisteskranke zusammen mit anderen Gegnern des faschistischen Systems getötet. Die Rezeption der Binding/Hoche-Schrift von 1920 über die Freigabe der Tötung «lebensunwer- 
ten» Lebens in der italienischen wissenschaftlichen Literatur war dem Referenten nicht bekannt. M. Ferro schilderte unter dem Titel: «Bilder der Schizophrenien von Griesinger bis Kraepelin» die engen wissenschaftlichen Kontakte zwischen der deutschsprachigen - insbesondere auch schweizerischen - Psychiatrie und Vertretern der italienischen Psychiatrie. Die historische Entwicklung des Schizophrenie-Konzepts beschrieb W. Schmitt. Er wies nach, dass heute kein einheitliches Konzept des Begriffes «Schizophrenie» existiert. Transkulturelle Ansätze bedachte G. Koptagel-Ilal in «Licht und Schatten in der Vergangenheit und in der gegenwärtigen Seelenheilkunde im Mittelosten». Auffällig in der modernen Türkei sei die Tendenz, dass alte Heilweisen für den politischen Zweck der Islamisierung missbraucht werden. Konzeptionen der Allgemeinbevölkerung über Psychiatrie und das Psychisch-krank-Sein berichtete C. Müller nach Ergebnissen von Untersuchungen im Abstand von 30 Jahren. Als Fortschritt wertete Müller den Befund, dass nach einer starken Abwehr und Abwertung des psychisch Kranken in früheren Jahren jetzt ein Gefühl der Ratlosigkeit bei der Bevölkerung aufgetreten sei, das zur Hoffnung Anlass gebe, dass ein Wandel der öffentlichen Meinung zur Psychiatrie eine Perspektive habe.

Als Ehrengast der Tagung konnte die «Internationale Arbeitsrunde zur Geschichte der Seelenheilkunde» Alice Riccardi von Platen-Hallermund begrüssen, die als Psychoanalytikerin und Gruppenanalytikerin seit Jahren in Rom lebt. Bekannt ist Frau von Platen-Hallermund als Mitarbeiterin der von Alexander Mitscherlich geleiteten Ärzte-Komission, die 1946 die Nürnberger Ärztekongresse beobachtete. Sie hat ihre Erfahrungen im Prozess in dem Buch «Die Tötung von Geisteskranken in Deutschland» 1947 vorgestellt.

Um den wissenschaftlichen Austausch zwischen italienischen und deutschen Kollegen zu fördern, wurde in Rom eine Deutsch-italienische, Italienisch-deutsche Arbeitsrunde gegründet, die sich regelmässig zu wissenschaftlichen Gesprächen abwechselnd in Deutschland und Italien trifft. Ansprechpartner in Deutschland ist Frau Dr. Hinnental Mandelli, in Italien Professor Lalli, Rom. 1998 wird die 7. Tagung der «Internationalen Arbeitsrunde zur Geschichte der Seelenheilkunde» voraussichtlich in Wien stattfinden. Das Tagungsthema ist noch offen. Vorgeschlagen wurde, über die Rolle des Psychiaters bzw. über den Begriff der Konstitution oder über Pathobiographien nachzudenken. Die Vorträge werden in der Reihe «Wissenschaftliche Beiträge zur Geschichte der Seelenheilkunde» veröffentlicht. Herausgeber G. Wahl/W. Schmitt, Verlag kommunikative Medien und Medizin, 88427 Reichenbach. Band 1: Vom Nutzen und Nachteil der Historie, 1994 (vergriffen). Band 2: Heilen - Verwahren - Vernichten» (im Mai 1997 erschienen). Band 3 behandelt das Thema Suizid, Band 4 Hysterie und Besessenheit, 
Band 5 Fremdbestimmung - Selbstbestimmtheit. Die Vorträge des römischen Treffens vom Mai 1997 werden im Band 6 Krankheitsbilder - Krankheitskonzepte (geplant ist eine deutsche und eine italienische Ausgabe) veröffentlicht.

G. Wahl

\section{Arbeitskreis Alte Medizin}

Zum 18. Treffen des Arbeitskreises Alte Medizin am Sonntag, dem 21. Juni 1998, ergeht Einladung ins Medizinhistorische Institut der Johannes Gutenberg-Universität Mainz, Am Pulverturm 13 (Untergeschoss). Anmeldungen für Vorträge und Mitteilungen richte man samt einer Zusammenfassung baldmöglichst - spätestens bis zum 15. März 1998 - an das Medizinhistorische Institut, Universitätsklinikum, D-55101 Mainz, Fax (+49) 6131/17 66 82, e-mail:kdfisch@goofy.zdv.uni-mainz.de. Meldungen zurTeilnahme sind auch kurzfristig möglich. Das endgültige Programm wird im April zum Versand kommen und geht neuen Interessenten auf Anforderung zu.

Klaus-Dietrich Fischer

\section{History of Science and Technology in Education and Training in Europe.}

Conference organised by the University Louis Pasteur Thursday, June 25 and Friday June 26, 1998, Strasbourg on behalf of ALLEA (All European Academies) in collaboration with the European Commission European Science and Technology Forum, DG XII

The history of science and technology is attracting growing attention in Europe. As a discipline of research, it can rely on strong intellectual traditions and on a reasonable level of human and material resources. In all European countries, the feeling is developing, however, that the history of science, technology and medicine does not play the significant role it should in education and training. It seems timely to review the present and possible role of the history of science and technology in education and training in Europe and to identify actions to be untertaken in order to improve the situation in this field. 


\section{Participants:}

Professional historians of science and technology; scientists, physicians, engineers and representatives of the humanities; representatives of government, media and industry.

Scientific Commitee:

(provisional list): Michel André (Brussels), Michel Blay (Paris), Jean Bottin (Paris), Paul Brouzeng (Paris), Sir Alan Cook (Cambridge), Salustiano Del Campo (Madrid), Claude Debru (Strasbourg), Wolfgang Eckart (Heidelberg), Robert Fox (Oxford), Tore Frängsmyr (Uppsala), Robert Halleux (Liège), Jan Janko (Prag), Walter Kaiser (Aachen), Juozas Krikstopaitis (Vilnius), Oivind Larsen (Oslo), Michel Meulders (Louvain la Neuve), John North (Groningen), Emmanuel Poulle (Paris), Christoph Scriba (Hamburg), Raffaella Simili (Bologna), Ferenc Szabadvary (Budapest), Inna Vartanian (St. Petersburg).

\section{Contact:}

Prof. Claude Debru, Centre Européen d'Histoire de la Médecine, Faculté de Médecine, 4 rue Kirschleger, 67085 Strasbourg Cedex France.

\section{Simposio Ciencia y Técnica en el 98: entre la liberación y el desastre VI Simposio de Enseñanza e Historia de la Ciencias}

Jaca, 24-28 de junio de 1998, Seminario de Historia de la Ciencia, Facultad de Ciencias (Matemáticas), Universidad de Zaragoza, Ciudad Universitaria, 50009 Zaragoza

\section{Neuerscheinung Oktober 1997:}

Phantastische Lebensräume, Phantome und Phantasmen.

Aufsätze des Zürcher Symposions der Schweizerischen Gesellschaft für Geschichte der Medizin und der Naturwissenschaften vom 10. und 11. Oktober 1996, herausgegeben von Hans-Konrad Schmutz, Marburg 1997.

254 Seiten, 50 Abb., engl. Broschur, DM 38.-, SFr 36.-, ISBN 3-925347-45-3. 
Inhalt: Hans-Konrad Schmutz, Randnotizen; Karl S. Guthke, Alptraum und Utopie. Extraterrestrische Welten von Galilei bis zur Goethezeit; Cornelius Steckner, Phantastische Belege oder phantastische Lebensräume? Fabelwesen in frühneuzeitlichen Naturalienkabinetten und Museen; Nicolaas Rupke, Paradise and the Notion of a World Centre, from the Physico-theologians to the Humboldtians; Peter Wegmann, Gefürchtet - gejagt - im Bilde gebannt: Phantastische Bewohner entlegener Lebensräume in der Kunst der Renaissance und des Barock; Véronique Dasen, Des Molionides à Janus: les êtres à corps ou à parties multiples dans l'Antiquité classique; Andrea Carlino, Strasni corpi. Come farsi una ragione dei monstri nel XVI secolo; Beat Rüttimann, Von Monstren und phantastischen Menschen; Urs Leo Gantenbein, Am Scheideweg zwischen Fiktion und Realität: Das Phantastische in Conrad Gessners Naturbetrachtung; Renato G. Mazzolini, L'illusione incomunicabile: il declino mocroscopia tra Sei e Settecento; Armin Geus, Anthropogenie und Menschenzucht. Biologistische Utopien im 19. und 20. Jahrhundert; Mario Wiesendanger, The Little Man in the Brain, Alchemy, Mandrake Roots, Mental Faculties, an Phrenology.

Bestellungen direkt an den Verlag: Basilisken-Presse, Postfach 561, D-35017 Marburg/Lahn. Telefon $06421 \cdot 15188$. 